\title{
Coherent Control of Two Nuclear Spins Using the Anisotropic Hyperfine Interaction
}

\author{
Yingjie Zhang, ${ }^{1,2}$ Colm A. Ryan, ${ }^{1,2}$ Raymond Laflamme, ${ }^{1,2,3}$ and Jonathan Baugh ${ }^{1,2,4, *}$ \\ ${ }^{1}$ Institute for Quantum Computing, University of Waterloo, Waterloo, Ontario, N2L 3G1, Canada \\ ${ }^{2}$ Department of Physics and Astronomy, University of Waterloo, Waterloo, Ontario, N2L 3G1, Canada \\ ${ }^{3}$ Perimeter Institute for Theoretical Physics, Waterloo, Ontario, N2J 2W9, Canada \\ ${ }^{4}$ Department of Chemistry, University of Waterloo, Waterloo, Ontario, N2L 3G1, Canada
}

(Received 14 July 2011; published 17 October 2011)

\begin{abstract}
We demonstrate coherent control of two nuclear spins mediated by the magnetic resonance of a hyperfine-coupled electron spin. This control is used to create a double-nuclear coherence in one of the two electron spin manifolds, starting from an initial thermal state, in direct analogy to the creation of an entangled (Bell) state from an initially pure unentangled state. We identify challenges and potential solutions to obtaining experimental gate fidelities useful for quantum information processing in this type of system.
\end{abstract}

DOI: 10.1103/PhysRevLett.107.170503

PACS numbers: 03.67.Lx, 76.70.Dx

Introduction.-Solid-state spin systems are interesting candidates for quantum information processing: the small systems explored in the lab today are excellent test beds for the ideas of quantum control and quantum error correction, and it may be possible to reach enough qubits for nontrivial quantum computations or to integrate these systems into useful hybrid devices for quantum communications [1] or quantum sensors [2]. The past two decades have seen much progress in the high-fidelity control of small quantum processors realized by nuclear magnetic resonance $[3,4]$, electron spin resonance (ESR) [5-9], and electron-nuclear double resonance [10-13]. These experiments have served as benchmarks for experimentally attainable gate fidelities [14] and have spurred the development of robust quantum control methods $[15,16]$. Hybrid electron-nuclear spin systems make it possible to exploit the strengths of each type of spin: electron spin for initialization, readout, and control and nuclear spin for long storage and coherence times $[12,13,17]$. In particular, it is advantageous to use the electron spin as an actuator to gain full control of the system's spin dynamics via the anisotropic part of the hyperfine interaction $[5,18]$. Since the hyperfine interaction to nearby nuclei can be of order $1-100 \mathrm{MHz}$, fast electron-nuclear and nuclear-nuclear gates can be realized by this approach. Several prior studies have demonstrated coherent control of a one electron + one nuclear spin system using a modulated microwave field in concert with anisotropic coupling $[5,7,8]$. In this work, we demonstrate an entangling gate between two nuclear spins fully mediated by control of the electron spin. This is an important first step towards achieving efficient control of hybrid electron-nuclear spin systems of interest for quantum information processing.

Experiment.-The spin system employed here is based on the stable radical of malonic acid in the solid state $[5,7,8,10]$ with an additional ${ }^{13} \mathrm{C}$ labeling. X-ray irradiation removes a proton from the methylene group, leaving behind an unpaired $\pi$ electron. The electron spin has $g$ factor $g=1.9843$ and couples via the Fermi contact and dipolar hyperfine interactions to the remaining methylene proton and to the ${ }^{13} \mathrm{C}$-labeled methylene carbon. The tensors describing the ${ }^{1} \mathrm{H}$ and ${ }^{13} \mathrm{C}$ hyperfine interactions were reported in Ref. [19]. Pulsed electron spin resonance was performed at an $X$-band microwave frequency of $9.1875 \mathrm{GHz}$ at room temperature on a home-built spectrometer. Numerically derived optimal control pulses were partially corrected for the finite resonator bandwidth and other pulse imperfections by installing a pickup antenna near the resonator and adjusting the input pulse until the measured pulse best matched the desired waveform. Additional experimental details may be found in the Supplemental Materials [20].

The secular internal spin Hamiltonian of the one electron + two nuclear spin system is given by

$$
\mathcal{H}=\Omega_{e} S_{z}+\omega_{H} I_{z}^{H}+\omega_{C} I_{z}^{C}+S_{z} \otimes \sum_{k \in C, H}\left(A_{k} I_{z}^{k}+B_{k} I_{x}^{k}\right)
$$

with the component of electron spin $S_{z}$ along the external field direction $\hat{z}$, nuclear spin operators $\mathbf{I}^{k}$, and where $\Omega_{e}$, $\omega_{H}$, and $\omega_{C}$ are the electron, ${ }^{1} \mathrm{H}$, and ${ }^{13} \mathrm{C}$ Zeeman frequencies, respectively, and $\left\{A_{k}, B_{k}\right\}$ are the four hyperfine coefficients. The small nuclear-nuclear dipolar coupling is neglected. In this system, $\Omega_{e} \gg A_{k}, B_{k}>\omega_{H}>\omega_{C}$, and the primary orientation dependence of the Hamiltonian is due to the hyperfine coefficients. Each nuclear spin has an anisotropic term $B S_{z} I_{x}$ that couples the longitudinal component of electron spin to a transverse component of nuclear spin. In combination with the nuclear Zeeman term, this causes the nuclear spin quantization axes to be dependent on the electron spin state and in general to be noncollinear with the external magnetic field (details on exploiting the anisotropic part of the hyperfine interaction are given in the Supplemental Materials [20]). We may 
choose logical qubit states of the nuclear two-spin system to be the energy eigenstates in either of the two manifolds defined by the electron spin-up or down; for example, the logical ${ }^{1} \mathrm{H}$ qubit states in the electron $|\uparrow\rangle$ manifold are

$$
\begin{aligned}
& \left|0_{H}\right\rangle=\cos \left(\theta_{\uparrow}^{H} / 2\right)\left|\uparrow_{H}\right\rangle-\sin \left(\theta_{\uparrow}^{H} / 2\right)\left|\downarrow_{H}\right\rangle, \\
& \left|1_{H}\right\rangle=\sin \left(\theta_{\uparrow}^{H} / 2\right)\left|\uparrow_{H}\right\rangle+\cos \left(\theta_{\uparrow}^{H} / 2\right)\left|\downarrow_{H}\right\rangle,
\end{aligned}
$$

where $\left|\uparrow_{H}\right\rangle$ and $\left|\downarrow_{H}\right\rangle$ are the nuclear eigenstates of the Zeeman interaction and $\theta_{\uparrow}^{H}=\tan ^{-1}\left(\frac{-B_{H}}{2 \omega_{H}+A_{H}}\right)$ is the angle between the proton quantization axis and the external field direction when the electron spin is up. Analogous expressions apply for the carbon spin states with angle $\theta_{\uparrow}^{C}$ and for the electron spin-down manifold with angles $\theta_{\downarrow}^{H, C}=$ $\tan ^{-1}\left(\frac{-B_{H, C}}{-2 \omega_{H, C}+A_{H, C}}\right)$. An allowed ESR transition, i.e., one that does not change the nuclear spin states in the limit of vanishing anisotropic terms, can be driven at a rate $\omega_{1} \cos \left(\frac{\theta_{\uparrow}-\theta_{\downarrow}}{2}\right)$ by a resonant microwave field of amplitude $\omega_{1}$. A forbidden transition, i.e., one involving one or more nuclear spin flips, is driven at a rate $\omega_{1} \sin \left(\frac{\theta_{1}-\theta_{\downarrow}}{2}\right)$. In our system, the forbidden transitions involving ${ }^{13} \mathrm{C}$ spin flips are strongly suppressed relative to the ${ }^{1} \mathrm{H}$ forbidden transitions, since the ${ }^{13} \mathrm{C}$ Zeeman frequency is very small in comparison with its secular hyperfine coefficient, $\left|A_{C}\right| \gg$ $\left|2 \omega_{C}\right|$.
The Hamiltonian parameters obtained for the crystal orientation used in these experiments are listed in the Table of Fig. 1(a).

For the crystal orientation we used, the field-swept ESR spectrum and the three-pulse electron-spin-echo envelope modulation (ESEEM) data are shown in Figs. 1(b) and $1(\mathrm{c})$, respectively. Note that only the ${ }^{1} \mathrm{H}$ transition frequencies appear in the ESEEM measurement due to the strong suppression of the forbidden transitions that involve the ${ }^{13} \mathrm{C}$ nucleus. The ESEEM spectra provide about 2 orders of magnitude better frequency precision than the field-swept due to the much longer nuclear $T_{2}^{*}$ time scales. The ${ }^{1} \mathrm{H}$ ESEEM frequencies are combined with the best-fit frequencies to the field-swept data, and, together with the known ${ }^{1} \mathrm{H}$ and ${ }^{13} \mathrm{C}$ hyperfine tensors, a most likely spin Hamiltonian is obtained. With this estimated Hamiltonian, optimal control pulses are numerically derived to perform the experiment described below; this experiment is equivalent to a "targeted" ESEEM that excites a double-nuclear coherence, giving us direct information about the ${ }^{13} \mathrm{C}$ Hamiltonian parameters and further refining the Hamiltonian estimate [21].

To demonstrate coherent control of the nuclear spin states, we implement an entangling quantum gate, for example, the operation that maps $|01\rangle \rightarrow \frac{|01\rangle \pm|10\rangle}{\sqrt{2}}$. The experiment is laid out schematically in Fig. 2(b). Starting from the thermal state deviation density matrix $\rho_{0}=S_{z}$, a selective $\pi$ pulse is applied to invert the population of the (a)

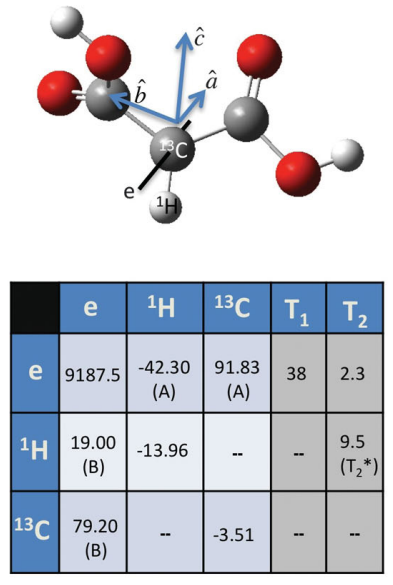

(b)

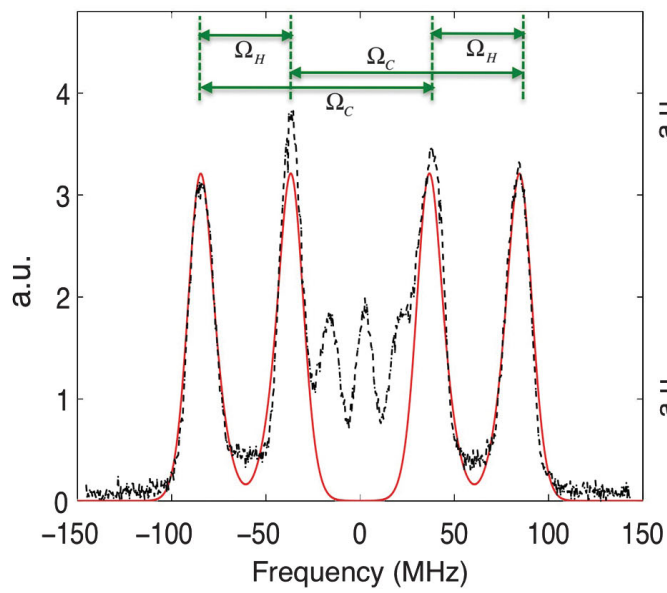

(c)

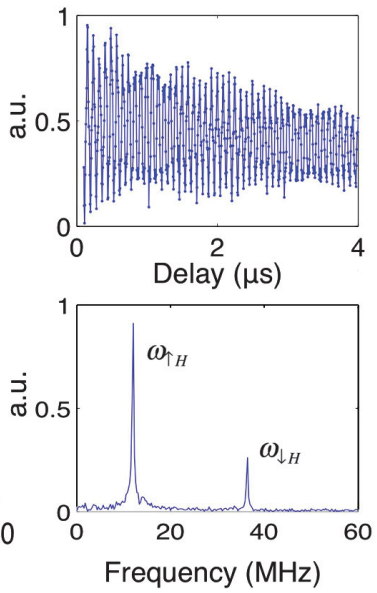

FIG. 1 (color). (a), upper: Schematic of the electron- ${ }^{13} \mathrm{C}-{ }^{1} \mathrm{H}$ system on the malonic acid radical. The electron occupies a $\pi$ orbital oriented along the $\hat{a}$ axis, perpendicular to the ${ }^{13} \mathrm{C}-{ }^{1} \mathrm{H}$ bond along the $\hat{c}$ axis of the Cartesian $(a, b, c)$ coordinate system shown $(\hat{b}$ and $\hat{c}$ are in the plane of the three carbon atoms). (a), lower: Table of Hamiltonian parameters for the crystal orientation used in this work, with external magnetic field direction $\hat{B_{0}}=(-0.55,0.27,0.79)$. Larmor frequencies appear along the diagonal, and hyperfine coupling coefficients are off-diagonal, with all frequencies in MHz. The two columns on the far right list observed $T_{1}$ and $T_{2}$ relaxation times in microseconds. (b) Field-swept ESR spectrum with experimental data (dotted black line) and best-fit simulation (solid red line). Additional peaks near the central region of the spectrum are due to another defect present in the crystal and may be ignored. The ${ }^{13} \mathrm{C}$ and ${ }^{1} \mathrm{H}$ splittings are $\Omega_{C}=122 \mathrm{MHz}$ and $\Omega_{H}=48 \mathrm{MHz}$, respectively. (c) Upper panel shows three-pulse ESEEM data recorded with hard pulses resonant with the transition at $+37 \mathrm{MHz}$; in the lower panel, its Fourier transform shows the two ${ }^{1} \mathrm{H}$ transition frequencies $\omega_{\uparrow H}=11.99 \pm 0.01 \mathrm{MHz}$ and $\omega_{\downarrow H}=36.35 \pm 0.04 \mathrm{MHz}$ corresponding to electron spin-up and spin-down states, respectively. 
transition at $\Omega_{e}+37 \mathrm{MHz}$, followed by a $0.8 \mu$ s optimal control gradient ascent pulse engineering (GRAPE) [15] pulse designed to perform a $\pi / 2$ rotation in the submanifold of the two nuclear states labeled in Fig. 2(a) by the $22 \mathrm{MHz}$ transition; the coherence between these two states is a double-nuclear coherence that behaves analogously to a pure state $\frac{|01\rangle+e^{i \phi}|10\rangle}{\sqrt{2}}$. Note that the latter operation could also be accomplished with an additional radio frequency (RF) channel to directly drive the nuclear transition (electron-nuclear double resonance) but would require at least several microseconds with typically available RF powers, as well as the complication of an additional RF interface. Subsequent to the selective $\pi$ (inversion) pulse, the ideal state of the system is

$$
\begin{aligned}
\rho= & -|\downarrow\rangle\langle\downarrow| \otimes\left(\mathbf{E}_{0}^{H} \otimes \mathbf{E}_{0}^{C}+\mathbf{E}_{1}^{H} \otimes \mathbf{E}_{0}^{C}-\mathbf{E}_{0}^{H} \otimes \mathbf{E}_{1}^{C}+\mathbf{E}_{1}^{H} \otimes \mathbf{E}_{1}^{C}\right) \\
& +|\uparrow\rangle\langle\uparrow| \otimes\left(\mathbf{E}_{0^{\prime}}^{H} \otimes \mathbf{E}_{0^{\prime}}^{C}+\mathbf{E}_{1^{\prime}}^{H} \otimes \mathbf{E}_{0^{\prime}}^{C}-\mathbf{E}_{0^{\prime}}^{H} \otimes \mathbf{E}_{1^{\prime}}^{C}+\mathbf{E}_{1^{\prime}}^{H} \otimes \mathbf{E}_{1^{\prime}}^{C}\right),
\end{aligned}
$$

where $\mathbf{E}_{m}^{j}=|m\rangle\langle m|$ is the density matrix corresponding to the energy eigenstate $|m\rangle(m \in 0,1)$ for nuclear spin $j$ and we label the eigenstates in the spin-up manifold by $\left|m^{\prime}\right\rangle$. The GRAPE pulse performs the following transformation in the spin-down manifold:

$$
\mathbf{E}_{1}^{H} \otimes \mathbf{E}_{0}^{C}-\mathbf{E}_{0}^{H} \otimes \mathbf{E}_{1}^{C} \Rightarrow|-\rangle\langle-|-|+\rangle\langle+|,
$$

where $| \pm\rangle=\frac{\left|0_{H} 1_{C}\right\rangle \pm\left|1_{H} 0_{C}\right\rangle}{\sqrt{2}}$ and the pulse acts as the identity
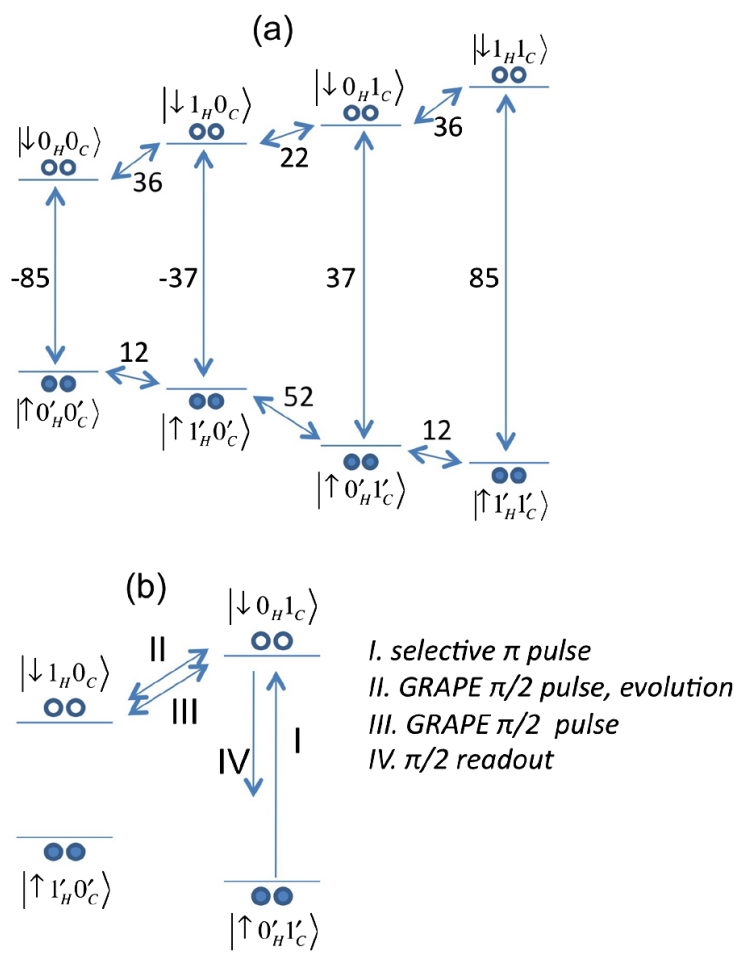

FIG. 2 (color online). (a) Energy level diagram for the threespin system showing transition frequencies (in $\mathrm{MHz}$ ) between eigenstates. $|\uparrow\rangle$ and $|\downarrow\rangle$ label the electron manifolds. Note that the nuclear quantization axes depend on the electron spin state, so that nuclear eigenstates in the two electron spin manifolds are different. (b) Schematic of the experimental sequence for creating and detecting double-nuclear coherence in the electron spindown manifold. Steps 1 and 4 refer to driving direct electronic transitions, while steps 2 and 3 involve an operation similar to a Hadamard gate (labeled "GRAPE $\pi / 2$ pulse") between the two nuclear sublevels $|01\rangle$ and $|10\rangle$. operator on all other terms. The terms on the right side of Eq. (5) evolve during a free evolution period $\tau$ as

$$
\begin{aligned}
& \cos \left[\left(\omega_{\downarrow C}-\omega_{\downarrow H}\right) \tau\right](|-\rangle\langle-|-|+\rangle\langle+|) \\
& \quad+\sin \left[\left(\omega_{\downarrow C}-\omega_{\downarrow H}\right) \tau\right](|+\rangle\langle-|+|-\rangle\langle+|),
\end{aligned}
$$

which is equivalent to precession of the pure state $\frac{\left|0_{H} 1_{C}\right\rangle+e^{-i \tau\left(\omega_{I} C^{\left.-\omega_{I H}\right)}\left|1_{H} 0_{C}\right\rangle\right.}}{\sqrt{2}}$ due to the internal Hamiltonian. Applying the GRAPE pulse again reverses the transformation in Eq. (5), so that the diagonal terms in the electron spin-down manifold $(|\downarrow\rangle\langle\downarrow|)$ become

$$
\begin{aligned}
& \mathbf{E}_{0}^{H} \otimes \mathbf{E}_{0}^{C}+\cos \left[\left(\omega_{\downarrow C}-\omega_{\downarrow H}\right) \tau\right]\left(\mathbf{E}_{1}^{H} \otimes \mathbf{E}_{0}^{C}-\mathbf{E}_{0}^{H} \otimes \mathbf{E}_{1}^{C}\right) \\
& \quad+\mathbf{E}_{1}^{H} \otimes \mathbf{E}_{1}^{C} .
\end{aligned}
$$

To perform readout, a selective $\pi / 2$ pulse is applied on the electron transition corresponding to the nuclear spin term $\mathbf{E}_{0}^{H} \otimes \mathbf{E}_{1}^{C}$. The observable is the electron spin $\hat{z}$ magnetization on this transition, and it can be shown [20] that the normalized signal as a function of delay $\tau$ is simply

$$
S(\tau)=\frac{1+\cos \left[\left(\omega_{\downarrow C}-\omega_{\downarrow H}\right) \tau\right]}{2} .
$$

The GRAPE pulse used in this experiment had a unitary fidelity of $98 \%$ on the ideal system, i.e., without including ensemble inhomogeneity effects (inhomogeneities of the dc and microwave magnetic fields throughout the sample), electron spin dephasing $\left(T_{2 e}\right)$, or other error sources such as the finite bandwidth of the ESR resonator and pulse imperfections [22]. When realistic $T_{2 e}$ and dc field inhomogeneity $\left(T_{2 e}^{*}\right.$ linewidth) are included in the simulation, the fidelity drops to $68 \%$, with each factor contributing roughly equal amounts to the error (note that the GRAPE pulse duration is about $1 / 3$ of $T_{2 e}$ in the present system). Spatial inhomogeneity of the microwave field and other pulse imperfections reduce the actual fidelities even further.

The experimental results are summarized in Fig. 3. Figure 3(a) shows the $22 \mathrm{MHz}$ modulation of the readout echo signal due to the evolution of the double-nuclear coherence in the electron spin-down manifold. Simulated data are also presented for comparison (blue dashed line); these were calculated at the same discrete time points as 
(a)

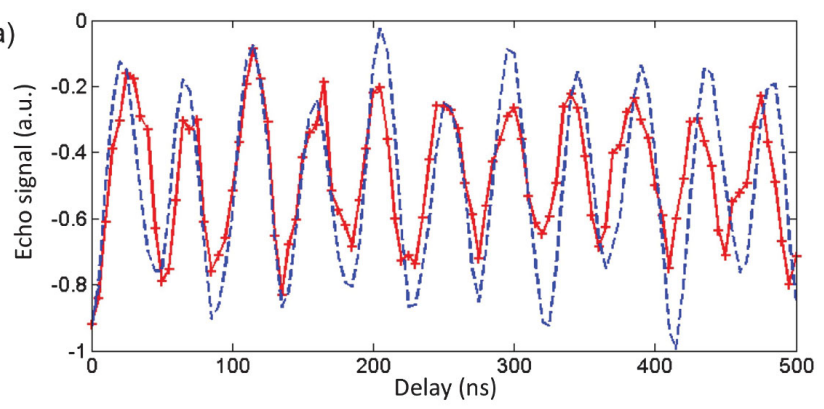

(b)
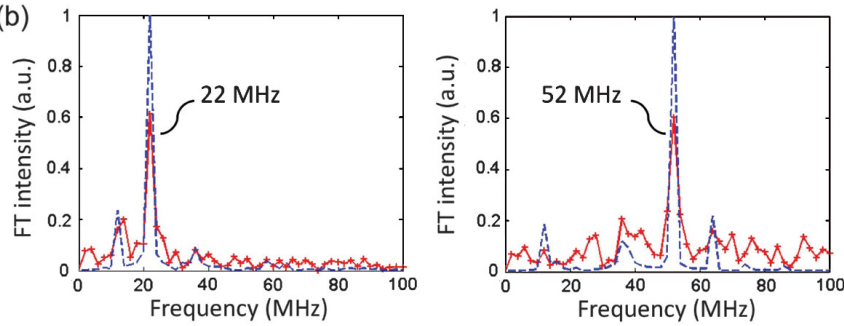

FIG. 3 (color). (a) The double-nuclear coherence echo signal versus delay time in experiment (red crosses, solid line) and simulation (blue dashed line) for the $22 \mathrm{MHz}$ transition in the electron spin-down manifold. (b) The Fourier transform of the oscillatory signal for the $22 \mathrm{MHz}$ transition experiment (left) and for the analogous experiment performed on the $52 \mathrm{MHz}$ transition in the electron spin-up manifold (right).

the real data and include the electron $T_{2 e}$ as well as the ensemble inhomogeneity $\left(T_{2 e}^{*}\right)$ effects. The Fourier transforms of the simulated and experimental data are both strongly peaked at $22 \mathrm{MHz}$ as expected, shown in the left panel of (b). The experiment was also carried out on the $52 \mathrm{MHz}$ transition in the electron spin-up manifold using a different GRAPE pulse, with results shown in the right panel of (b). For both experiments, the modulation amplitude of the measured signal is about 1.6 times smaller than that predicted by simulation. The best fits to the experimental data give frequencies $22.3 \pm 0.5 \mathrm{MHz}$ and $52.0 \pm$ $0.4 \mathrm{MHz}$ in excellent agreement with the estimated Hamiltonian. Note that a small amount of $12 \mathrm{MHz}$ modulation due to the ${ }^{1} \mathrm{H}$ transitions in the spin-up manifold is present in the $22 \mathrm{MHz}$ transition data, both in simulation and experiment. We emphasize that we have not been able to reproduce the 22 and $52 \mathrm{MHz}$ frequencies in any type of standard ESEEM experiment, including "matched" ESEEM [23] with soft pulses designed to favor excitation of those coherences.

Conclusion.-In summary, we have demonstrated experimentally that an electron can be used as an actuator to perform an entangling gate between two nuclear spins. This is a first step towards reaching high-fidelity, universal control of one electron $+N$-nuclear spin hyperfine-coupled systems using a single microwave field. Several factors limited the fidelity of coherent control achievable in this experiment. First, the bandwidth of the loop-gap resonator must cover the full spectral width so that all the system transitions can be driven efficiently. In our experiment, the $Q$ factor could only be spoiled to achieve a bandwidth $\approx 140 \mathrm{MHz}$, smaller than the full spectral width of $170 \mathrm{MHz}$; indeed, some features of the GRAPE pulses involving high-frequency components could not be adequately corrected with our pickup antenna feedback method [20]. Secondly, spoiling the $Q$ factor reduces the maximum Rabi frequency proportionately; when the Rabi frequency $\nu_{\text {Rabi }} \ll\left\|\mathcal{H}_{\text {rot }}\right\|$ where $\mathcal{H}_{\text {rot }}$ is the rotating-frame Hamiltonian, the system is still controllable, in principle, but requires pulses that are significantly longer than the nearly time-optimal pulses that can be found when $\nu_{\text {Rabi }} \geq\left\|\mathcal{H}_{\text {rot }}\right\|$. When the latter condition is satisfied, we find empirically that pulses with much better robustness to ensemble inhomogeneities can be obtained, and furthermore, efficient decoupling of the electron from the nuclear spin bath would be possible with hard pulses, allowing $T_{2 e}$ to be significantly lengthened $[7,9]$. The coherence times of the nuclei as well as the electron ultimately depend on the relaxation time $T_{1 e}$ of the electron, which dramatically increases at low temperatures $(T \ll 10 \mathrm{~K})$ in malonic acid [24] and in many other spin systems. We are presently working to extend these experiments to that regime and to utilize spin systems with narrower spectral widths. Finally, we remark that simulations support the theoretical result $[5,18]$ that it is possible to construct any desired unitary operator in the Hilbert space of the three-spin system. For example, control-NOT and SWAP gates acting on the two nuclei can be found numerically with similar durations and unitary fidelities as the pulse used in the experiments above, with the same constraints.

We would like to acknowledge fruitful discussions with D. G. Cory and technical assistance from J. Chamilliard, M. Ditty, and H. van der Heide. This work was supported by the Natural Sciences and Engineering Research Council of Canada and the Canada Foundation for Innovation.

*baugh@iqc.ca

[1] L. Childress, J.M. Taylor, A.S. Sørensen, and M. D. Lukin, Phys. Rev. Lett. 96, 070504 (2006).

[2] J. R. Maze, P. L. Stanwix, J.S. Hodges, S. Hong, J. M. Taylor, P. Cappellaro, L. Jiang, M. V. G. Dutt, E. Togan, and A. S. Zibrov et al., Nature (London) 455, 644 (2008).

[3] J. Baugh, J. Chamilliard, C. M. Chandrashekar, M. Ditty, A. Hubbard, R. Laflamme, M. Laforest, D. Maslov, O. Moussa, and C. Negrevergne et al., Phys. Canada 63, 197 (2007).

[4] D. G. Cory, R. Laflamme, E. Knill, L. Viola, T. Havel, N. Boulant, G. Boutis, E. Fortunato, S. Lloyd, and R. Martinez et al., Fortschr. Phys. 48, 875 (2000).

[5] J. S. Hodges, J. C. Yang, C. Ramanathan, and D. G. Cory, Phys. Rev. A 78, 010303 (2008).

[6] F. Jelezko, T. Gaebel, I. Popa, A. Gruber, and J. Wrachtrup, Phys. Rev. Lett. 92, 076401 (2004). 
[7] J. Du, X. Rong, N. Zhao, Y. Wang, J. Yang, and R. B. Liu, Nature (London) 461, 1265 (2009).

[8] G. Mitrikas, Y. Sanakis, and G. Papavassiliou, Phys. Rev. A 81, 020305 (2010).

[9] C. A. Ryan, J. S. Hodges, and D. G. Cory, Phys. Rev. Lett. 105, 200402 (2010).

[10] M. Mehring, J. Mende, and W. Scherer, Phys. Rev. Lett. 90, 153001 (2003).

[11] W. Scherer and M. Mehring, J. Chem. Phys. 128, 052305 (2008).

[12] S. Simmons, R. M. Brown, H. Riemann, N. V. Abrosimov, P. Becker, H.-J. Pohl, M. L. W. Thewalt, K. M. Itoh, and J. J. L. Morton, Nature (London) 470, 69 (2011).

[13] P. Neumann, N. Mizuochi, F. Rempp, P. Hemmer, H. Watanabe, S. Yamasaki, V. Jacques, T. Gaebel, F. Jelezko, and J. Wrachtrup, Science 320, 1326 (2008).

[14] C. A. Ryan, M. Laforest, and R. Laflamme, New J. Phys. 11, 013034 (2009).

[15] N. Khaneja, B. Heitmann, A. Spörl, H. Yuan, T. SchulteHerbrüggen, and S. J. Glaser, Phys. Rev. A 75, 012322 (2007).

[16] R. Nigmatullin and S. G. Schirmer, New J. Phys. 11, 105032 (2009).

[17] L. Jiang, J. S. Hodges, J. R. Maze, P. Maurer, J. M. Taylor,
D. G. Cory, P. R. Hemmer, R. L. Walsworth, A. Yacoby, and A. S. Zibrov et al., Science 326, 267 (2009).

[18] N. Khaneja, Phys. Rev. A 76, 032326 (2007).

[19] T. Cole and C. Heller, J. Chem. Phys. 34, 1085 (1961).

[20] See Supplemental Material at http://link.aps.org/ supplemental/10.1103/PhysRevLett.107.170503 for an overview of exploiting the anisotropic hyperfine interaction and additional experimental details.

[21] The initial Hamiltonian estimate was found to agree with the refined estimate to within $1.6 \%$, in the sense that $\left\|\mathcal{H}-\mathcal{H}^{\prime}\right\|<0.016\|\mathcal{H}\|$, where $\mathcal{H}$ and $\mathcal{H}^{\prime}$ are the original and refined estimates for the rotatingframe Hamiltonian.

[22] Note, however, that numerical optimization of the GRAPE pulse was performed over a discrete set of dc fields designed to roughly match the $T_{2 e}^{*}$ linewidth in order to improve the "robustness" of the pulse. Without this constraint, much higher fidelity pulses on the ideal system are easily found, but these pulses would perform much worse in the actual experiment.

[23] G. Jeschke, R. Rakhmatullin, and A. Schweiger, J. Magn. Reson. 131, 261 (1998).

[24] L. Dalton, A. Kwiram, and J. Cowen, Chem. Phys. Lett. 14, 77 (1972). 